\title{
POLÍTICAS UNIVERSITARIAS DE DIFUSIÓN DE LA INFORMACIÓN A TRAVÉS DE LA PROPIA WEB INSTITUCIONAL
}

\section{Mario Pérez-Montoro}

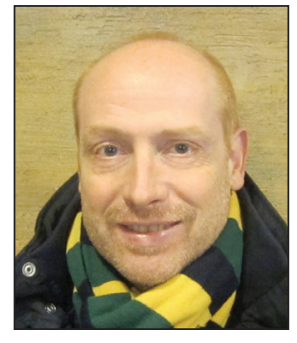

Mario Pérez-Montoro es doctor en filosofía y ciencias de la educación por la Universidad de Barcelona $(U B)$ y máster en organización de sistemas de documentación en la empresa por la Universidad Politécnica de Cataluña. Ha realizado estudios de postgrado en el Istituto di Discipline della Comunicazione de la Università di Bologna (Italia) y ha sido profesor visitante en el Center for the Study of Language and Information (CSLI) de la Stanford University, California, EUA, y en la School of Information de la UC Berkeley, California. Es profesor de la Facultad de Biblioteconomía y Documentación de la UB. Su investigación se centra en el ámbito de la arquitectura y la visualización de la información. Es autor del libro Arquitectura de la información en entornos web (Trea, 2010). http://orcid.org/0000-0003-2426-8119

Universitat de Barcelona, Departament de Biblioteconomia i Documentació Melcior de Palau, 140. 08014 Barcelona, España perez-montoro@ub.edu

\section{Resumen}

La difusión de la información se ha convertido en uno de los elementos críticos en los planes estratégicos de las instituciones universitarias. Inquietadas por su posición en los rankings internacionales que evalúan este tipo de instituciones, las universidades comienzan a preocuparse también por sus estrategias de difusión de información y sus webs institucionales. En este trabajo se abordan las funciones principales de las webs universitarias; el proceso de análisis, diseño e implementación en la creación de este tipo de sitios webs; los elementos mínimos que deben incluirse en una guía de estilo de web universitaria; y algunos de los principales estándares y referencias nacionales e internacionales de ese tipo de guías.

\section{Palabras clave}

Difusión de la información, Web universitaria, Guía de estilo web, Arquitectura de la información, Usabilidad, Accesibilidad, Universidad.

\section{Title: University policies on information dissemination through their institutional websites}

\section{Abstract}

Information dissemination has become a critical element of universities' strategic plans. Concerned about their position in the international rankings that evaluate this kind of institutions, universities also begin to worry about their information dissemination strategies and their institutional websites. This article addresses the main functions of the university website; the process of analysis, design and implementation in the production of this kind of websites; the minimum elements to be included in a university web style guide; and some key national and international style guide standards and references.

\section{Keywords}

Information dissemination, University web site, Web site style guidelines, Information architecture, Usability, Accessibly, University.

Pérez-Montoro, Mario (2014). "Políticas universitarias de difusión de la información a través de la propia web institucional”. El profesional de la información, marzo-abril, v. 23, n. 2, pp. 190-194.

http://dx.doi.org/10.3145/epi.2014.mar.12

\section{Introducción}

La difusión de la información se ha convertido en uno de los elementos críticos en los planes estratégicos de las instituciones universitarias.

Inquietadas por su posición en los rankings internacionales que evalúan este tipo de instituciones, las universidades co- mienzan a preocuparse también por sus estrategias de difusión de información a través de sus webs institucionales. Y se preocupan por esa difusión junto a otros indicadores de calidad más clásicos como el grado de integración laboral de sus egresados, el número de doctores integrados en sus plantillas de profesorado o el volumen y la calidad de la producción científica de sus investigadores. 
En este trabajo se abordan las funciones principales de las webs universitarias; el proceso de análisis, diseño e implementación en la creación de este tipo de sitios webs; los elementos mínimos que deben incluirse en una guía de estilo de web universitarias; y algunos de los principales estándares y referencias nacionales e internacionales de ese tipo de guías.

\section{Funciones de la web institucional}

En términos generales, la función principal de la web institucional de una universidad es la difusión de la información relacionada y generada en esa organización. Es posible desdoblar ese objetivo general en tres funciones básicas: comunicación, suministro de servicios y gestión del conocimiento.

La web institucional se presenta como el medio de comunicación entre los equipos directivos institucionales y la comunidad (estudiantes, profesorado y personal de administración) universitaria. En esta línea, en la web se ofrece desde la misión, historia y las cifras de la universidad, hasta su organización, titulaciones y edificios que la conforman. Por otro lado, a través de la web se suministran online también algunos de los principales servicios universitarios. Entre ellos destacan, por ejemplo, el campus virtual (para la implementación del modelo blended o híbrido (presencial con enriquecimiento virtual de enseñanza-aprendizaje), los servicios (biblioteca, derechos de autor o formación de usuarios, entre otros) relacionados con el crai (centro de recursos para el aprendizaje y la investigación) o el repositorio institucional, que difunde una parte importante de los documentos derivados de la actividad docente y la investigación. Por último, la web institucional contribuye a la gestión del conocimiento interno de la universidad (Davenport; Probst, 2002). Esa gestión se realiza a través de, entre otros, la intranet institucional o departamental, los espacios para la gestión de la investigación o el sistema de gestión del currículum de los docentes que permite la difusión y evaluación de la docencia y la investigación de sus profesores.

\section{Diseño}

Los cambios metodológicos introducidos en los últimos años dentro de la disciplina del diseño web recomiendan planificar e implementar un sitio web a partir de la realización de una serie de estudios de sus usuarios tipo. Este proceso acostumbra a desarrollarse a lo largo de tres fases diferenciadas: fase de análisis, fase de diseño y fase de implementación (Pérez-Montoro, 2010).

En la fase de análisis se hacen estudios a sus usuarios tipo para identificar las necesidades y los requerimientos informativos que va a cubrir el sitio web. En la fase de diseño se propone la estructura arquitectónica de la web a partir de esas necesidades y requerimientos identificados. Por úl- timo, la web se implementa y se somete a una serie de tests y pruebas que permitan comprobar que se han cubierto dichas necesidades y requerimientos.

En el caso de una web universitaria, el resultado de este proceso es un sitio institucional con una clara estructura arquitectónica. Por un lado, suele presentar un sistema de organización por audiencia (Morville; Rosenfeld, 2006) orientado a cinco tipos de usuarios: estudiantes en prospectiva, profesorado, investigadores, estudiantes y personal de administración. Por otro, se incluye un sistema de navegación constante, completado con otro local y otro contextual, que permite la exploración orientada de los principales contenidos incluidos en el sitio. También se incorpora un sistema de etiquetado sintonizado con esos cinco tipos de usuarios y un sistema de búsqueda que permite la recuperación de contenidos a partir de su indización. El diseño de la web se suele completar sometiéndola a análisis heurísticos ${ }^{1}$ de usabilidad $y$, como toda web institucional, introduciendo los cambios necesarios para que cumpla los estándares de accesibilidad que facilitan su uso por parte de visitantes con algún tipo de discapacidad.

En la web de la universidad se ofrece su misión, historia, cifras, organización, titulaciones, edificios...

\section{Guía de estilo web}

El proceso de diseño e implementación culmina con la redacción de la guía de estilo. El objetivo principal de este documento es recoger todos los aspectos y requisitos relacionados con la arquitectura, usabilidad y accesibilidad del sitio incluidos en su diseño para que los encargados de im- 


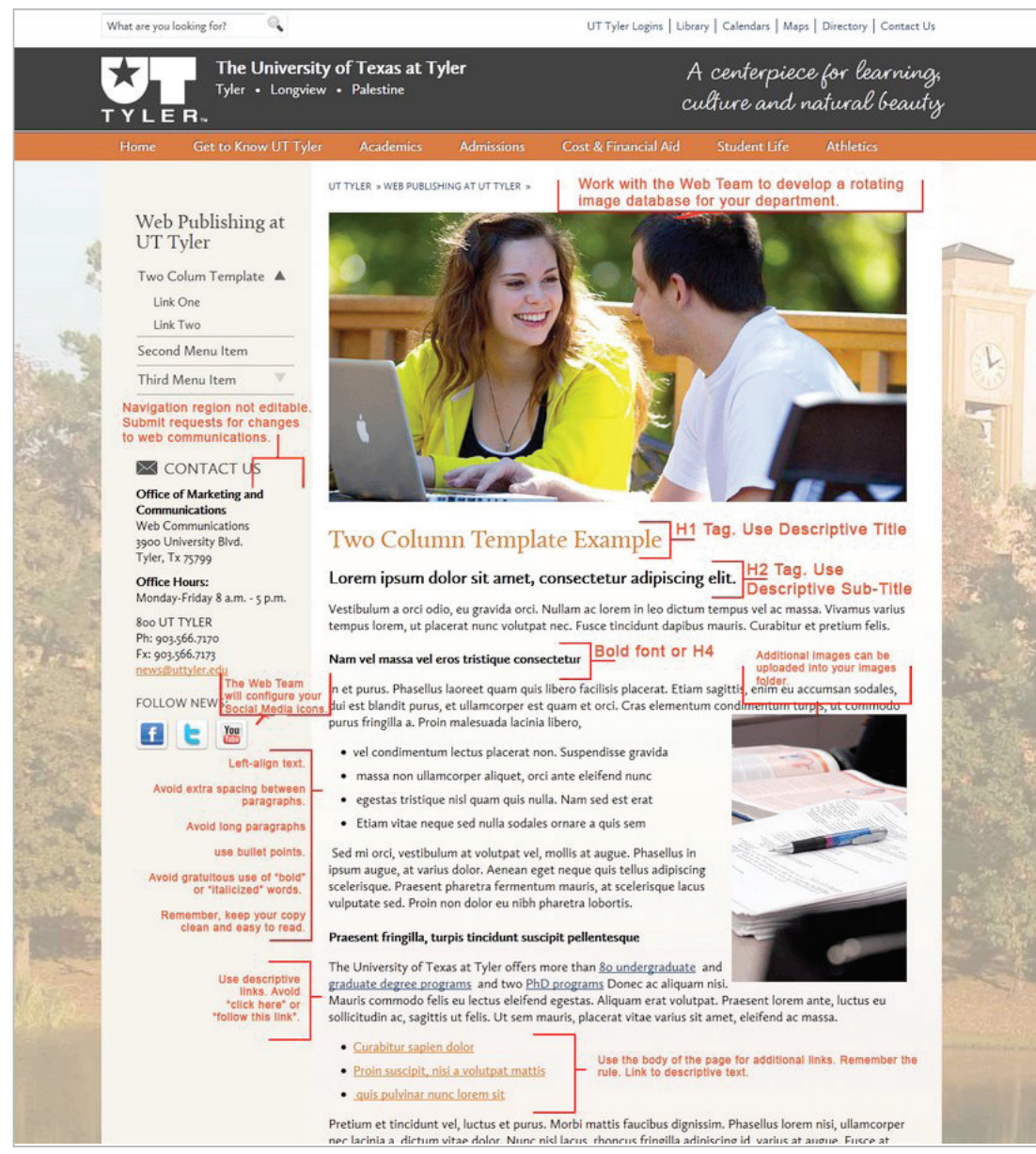

https://www.uttyler.edu/web/ou/training/styleguide.php
Sin embargo, en la guía de una web universitaria se deben incluir también otros elementos visuales y de estilo imprescindibles para la correcta y consistente implementación, actualización y ampliación futura.

En esta línea, es necesario recoger todos los elementos visuales relacionados con la imagen de marca institucional. Así, se debe ofrecer una galería de los diferentes logos y elementos de branding (en sus diferentes tamaños) de la universidad. Relacionado con esto, se debe describir también el tema del color y su uso tipográfico para cada uno de los tipos de contenido, y establecer la clase de cabecera, banners y titulares que se utilizarán.

Los aspectos visuales y estructurales de los elementos multimedia deben ser recogidos. Se debe presentar una guía de estilo, tamaños y proporciones de las fotografías que se puedan incorporar, sin descuidar la inclusión del vocabulario o galería de iconos de visualización (archivo, correo electrónico, enlace, carpeta, etc.) que se pueden utilizar. En la misma línea, se deben ofrecer las instrucciones sobre el uso, proporción, estilo y tecnologías utilizadas en la incorporación de gráficos y vídeos. plementar y mantener la web puedan hacerla evolucionar en un futuro respetando esos aspectos y garantizando así su correcto funcionamiento (Lynch; Horton, 2009).

No existe un único estándar aceptado a la hora de redactar y estructurar una guía de estilo web. Pero en el caso de las webs universitarias, es posible identificar unas recomendaciones y elementos mínimos que deben ser incluidos en un documento de este tipo para dotarlo de funcionalidad.

En este sentido, el documento debe recoger los objetivos comunicativos que se persiguen, dejando clara la tipología de sus usuarios y sus necesidades informativas. También debe describir la organización de sus contenidos, sus metadatos, y cuál es el proceso que debe seguirse en caso de la incorporación de nuevos contenidos e información. Debe incluirse también el ciclo de vida de sus contenidos, mostrando el papel y responsabilidades de todos los actores implicados en ese ciclo de vida. Se deben incorporar los libros de laboratorio derivados del proceso de análisis, diseño e implementación del sitio web, donde se recoja con detalles las acciones y decisiones tomadas a lo largo de todo ese proceso. El documento se completa con una biblioteca de los prototipos utilizados en el diseño (blueprints y wireframes), el repositorio de plantillas (cascading style sheets, CSS) descargables de cada uno de los modelos de contenido que conforman el sitio y la relación de todos los tests y pruebas realizadas a los usuarios involucrados en el proceso de desarrollo.
El texto incluido reclama también un tratamiento especial en la guía de estilo de una web universitaria. En ese sentido, la guía debe ofrecer un repositorio donde se muestren las tipografías y elementos textuales que se pueden utilizar en la creación de cada tipo de contenido. El tratamiento de los elementos textuales se completa ofreciendo las normas de creación de hipertextos (características tipográficas y de diseño de los literales), las parrilla de espaciados y distribución de unidades de contenido en cada tipo de página y la guía de estilo de las tablas que eventualmente se puedan incorporar al sitio web.

\section{El diseño de la web se suele completar sometiéndola a análisis heurísticos de usabilidad}

No podemos acabar de abordar los elementos mínimos que debe incluir la guía de estilo de una web universitaria sin recomendar dos cosas importantes. Por un lado, en la misma se deben recoger tanto ejemplos de buenas como de malas prácticas (errores a evitar) respecto a esos elementos tratados. Y, por otro, esta guía debe ser descargable (como una única unidad) desde la propia web universitaria para que pueda ser utilizada por los encargados de la actualización y desarrollo del sitio web. 


\section{Estándares y referencias}

Existe un larga lista de quías de estilo y directrices para el desarrollo de una página web universitaria, aunque sólo unas pocas son reconocidas como estándares y referencias por parte de los diseñadores. Destacan dos grandes grupos:

- las guías de webs gubernamentales;

- las de ciertas universidades de prestigio.

Las principales guías gubernamentales de referencia son anglosajonas. Concretamente, resaltan las que ofrece tanto el gobierno norteamericano (Usability. gov) como el británico (Guidelines for UK Government websites). Aunque existen guías de estilo ofrecidas también por otros países, como, por ejemplo, Web standards (New Zealand), Tasmanian Government web usability guidelines (Tasmania), Swedish national guidelines for public sector websites (Suecia) o Elmer (Noruega), entre otros. En el contexto latino y peninsular citaremos la Guía web de Chile, la Guía de usos y estilo en las redes sociales del Gobierno Vasco o la Guía de usos y estilo de la Generalidad de Cataluña.

Todas estas guías muestran una estructura muy similar. Ofrecen una serie de heurísticos de arquitectura de la información, usabilidad y accesibilidad, apoyados en evidencias y bibliografía científica, y un código de buenas y malas prácticas.

En el caso de las guías de estilo web universitarias, las más reconocidas se corresponden con instituciones académicas de prestigio como Yale University, Carnegie Mellon University, University of Texas o Columbia University. En el contexto nacional, muchas universidades españolas (Universidad del País Vasco, Universidad de Málaga, Universidad de Murcia o Universidad de Cádiz, entre otras) ofrecen también sus guías de estilo web.

Al igual que en el caso de las gubernamentales, estas guías comparten un patrón estructural muy similar. En ellas se ofrecen los principales elementos arquitectónicos, de usabilidad y de accesibilidad de las páginas, junto a una descripción exhaustiva de los atributos visuales (imagen de marca institucional, cabeceras, banners, titulares y elementos multimedia y textuales, entre otros) que conforman los contenidos del sitio.

\section{Nota}

1. El análisis heurístico ayuda a identificar problemas de usabilidad en el diseño de interfaces de usuario. Los evaluadores examinan la interfaz y juzgan su conformidad con los principios de usabilidad reconocidos. En general, los análisis heurísticos permiten determinar la susceptibilidad de un sistema hacia una amenaza particular o riesgo utilizando reglas de decisión y métodos de evaluación establecidos previamente. Se contrapone al análisis estadístico, que se basa en datos y estadísticas reales.

\section{Bibliografía}

Carnegie Mellon University. Marketing Communications. Web guidelines.

http://www.cmu.edu/marcom/brand-guidelines/print-webproducts/web/index.html

Columbia University. Web \& identity guidelines.

http://www.columbia.edu/content/web-identity-guidelines. html

Davenport, Thomas; Probst, Gilbert (eds.) (2002). Knowledge management case book. Munich: Verlag-Wiley.

Department of Premier and Cabinet. Office of eGovernment (State of Tasmania) (2012). Tasmanian Government web usability guidelines: version 1.4a. Hobart.

http://www.egovernment.tas.gov.au/_data/assets/ pdf_file/0008/78254/Web_Usability_Guidelines_V1.4a_ July_2012.pdf 
Generalitat de Catalunya. Departament de la Presidència (2010). Direcció General d'Atenció Ciutadana. Guia d'usos $i$ estil a les xarxes socials de la Generalitat de Catalunya. http://www.gencat.cat/xarxessocials/pdf/v1_guia_usos_ xarxa_cat.pdf

Gobierno de Chile. Guía web. http://www.guiadigital.gob.cl/guia-web

Gobierno Vasco. Presidencia (2011). Guía de usos y estilo en las redes sociales del Gobierno Vasco. Vitoria: Servicio Central de Publicaciones del Gobierno Vasco.

http://goo.gl/lqhHfF

Government Chief Information Officer (2013). Web publishing. New Zealand Government.

http://ict.govt.nz/guidance-and-resources/standardscompliance/web-publishing

Lynch, Patrick J.; Horton, Sarah (2009). Web style guide: Basic design principles for creating web sites. New Haven (CT): Yale University Press.

Norwegian Ministry of Trade and Industry (2006). Elmer 2: user interface guidelines for governmental forms on the Internet. http://www.brreg.no/eer/elmer2-english.pdf

Morville, Peter; Rosenfeld, Louis (2006). Information architecture for the World Wide Web. Sebastopol (CA): O'Reilly Media Inc.

Pérez-Montoro, Mario (2010). Arquitectura de la información en entornos web. Gijón: Trea.

The University of Texas at Austin. University Communications. Web publishing guidelines.

http://www.utexas.edu/brand-guidelines/web-guidelines
U.S. Department of Health and Human Services. Digital Communications Division (2014). Usability.gov: improving the user experience. Washington, D.C: U.S. Department of Health and Human Services.

http://www.usability.gov

UK Government (2008). Web guidelines.

http://goo.gl/PwH3wu

Universidad de Cádiz. Gabinete de Comunicación y Marketing (2010). Guía de estilo del portal UCA. Cádiz.

http://www.uca.es/recursos/doc/Unidades/Gab_Com_ Mark/465200059_19420109123.pdf

Universidad de Málaga. Manual de estilo web 2.0.

http://www.uma.es/media/files/GUIA_WEB.pdf

Universidad de Murcia. Guía de estilo del web de la Universidad de Murcia.

http://www.s/d.cu/galerias/pdf/sitios/vigilancia/guiaestilo-web.pdf

Universidad Politécnica de Valencia. Área de Sistemas de la Información y las Comunicaciones. Manual de estilos centros/servicios: guía de uso general para maquetación web. http://www.upv.es/entidades/ASIC/menu_urlc.htmI?/ entidades/ASIC/manuales/guia_estilos_upv.pdf

Verva - Swedish Administrative Development Agency (2008). Swedish national guidelines for public sector websites. http://arkiv.edelegationen.se/verva/upload/english/ swedish-guidelines-public-sector-websites.pdf

Yale University (2012). Yale web style guide.

http://www.yale.edu/web/styleguide

\title{
Anuario ThinkEPI 2013
}

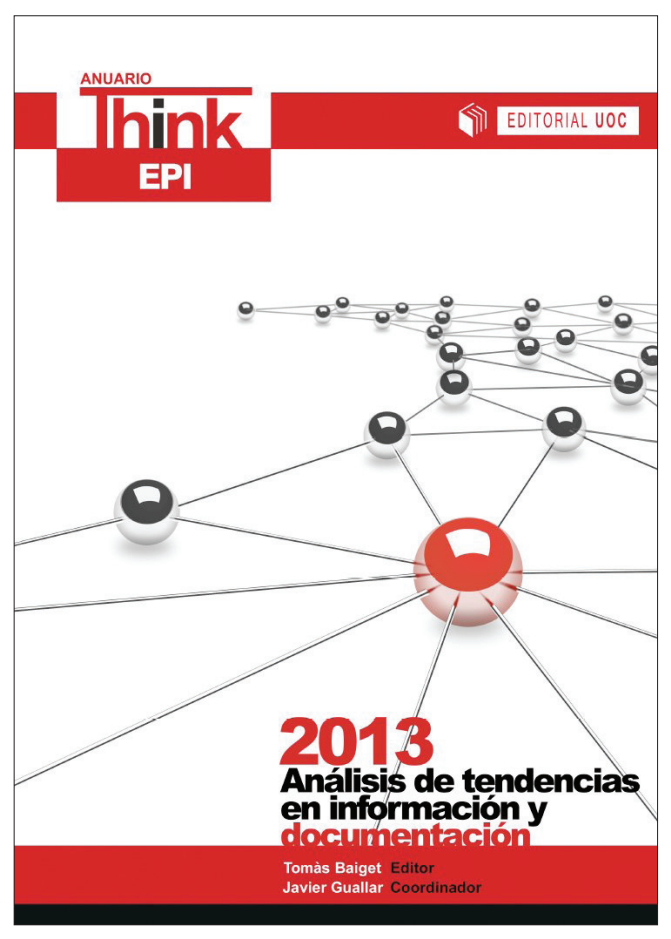

\section{2 páginas de análisis de tendencias en información, documentación y comunicación}

\author{
Formulario de compra: \\ http://www. \\ elprofesionaldelainformacion.com/ \\ suscripciones.php
}

\section{Información y pedidos:}

Isabel Olea

epi.iolea@gmail.com

+34608491521 